\title{
PROPIEDADES PSICOMÉTRICAS DE LA ESCALA DE CALIDAD DE VIDA DE BARNES Y OLSON EN ESTUDIANTES LIMEÑOS (ECVOB)
}

\section{PSYCHOMETRIC PROPERTIES OF THE OLSON AND BARNES QUALITY OF LIFE SCALE IN LIMA STUDENTS}

\author{
Mirian Pilar Grimaldo ${ }^{1}$, Jossué David Correa1, Diego Jara ${ }^{1}$, Ingrid Belu Cirilo² y Marivel Teresa Aguirre ${ }^{2}$ \\ ${ }^{1}$ Universidad Peruana de Ciencias Aplicadas. Perú \\ ${ }^{2}$ Universidad Nacional Federico Villarreal. Perú
}

\section{Abstract}

Quality of life refers to the way in which the person perceives their daily experience, considering in this process the social and cultural component. This implies that to know the perception of the quality of life it is necessary to consider in its measurement the scope of physical, psychological, social, environmental and personal health. Along these lines, one of the instruments that is limited to this approach is the Barnes and Olson Quality of Life Scale (ECVOB). Objective. The purpose of the study was to determine the validity based on the internal structure and reliability of the ECVOB in schoolchildren and university students in Lima. Method. The study design is instrumental. The Spanish version of the ECVOB was used on a sample of 1239 students from Lima between males (57\%) and females (43\%) aged between 14 and 26 years $\left(M_{\text {age }}=22.45 ; S D\right.$ age $\left.=3.75\right)$. The evidence of validity of the internal structure was evaluated through a confirmatory factor analysis. Results. The findings suggest a partial restructuring of the original seven-factor model proposed by Olson and Barnes. Conclusion. The new structure of the ECVOB is a valid and reliable measure in schoolchildren and university students in Lima.

Keywords: Quality of life, psychometrics, students, validity, reliability.

\section{Resumen}

La calidad de vida se refiere a la forma en que la persona percibe su vivencia cotidiana, considerando en ese proceso el componente social y cultural. Ello implica que para conocer la percepción de la calidad de vida es necesario considerar en su medición el ámbito de la salud física, psicológica, social, ambiental y personal. En esta línea uno de los instrumentos que se circunscribe a este enfoque es la Escala de Calidad de Vida de Barnesy Olson (ECVOB). Objetivo. El propósito del estudio fue determinar la validez basada en la estructura interna y la fiabilidad del ECVOB en escolares y universitarios de Lima. Método. El diseño del estudio es instrumental. Se utilizó la versión española de la ECVOB sobre una muestra de 1239 estudiantes limeños entre varones (57\%) y mujeres (43\%) de edades comprendidas entre 14 y 26 años ( $M_{\text {edad }}=22.45 ; D_{\text {edad }}=3.75$ ). La evidencia de validez de la estructura interna se evaluó a través de un análisis factorial confirmatorio. Resultados. Los hallazgos sugieren una reestructuración parcial del modelo original de siete factores propuesto por Olson y Barnes. Conclusión. La nueva estructura de la ECVOB es una medida valida y fiable en escolares y universitarios limeños.

Palabras clave: Calidad de vida, estudiantes, fiabilidad, psicometría, validez. 
La Organización Mundial de la Salud (OMS, 1998) considera a la calidad de vida como la forma a partir de la cual la persona percibe su vida, considerando el componente cultural; así como el sistema de valores, expectativas y normas, expresados en su vida cotidiana en relación con las actividades que realiza en el ámbito de la salud física, psicológica, relaciones sociales, factores ambientales y personales.

Por su parte, Diener (2006) asume que la calidad de vida es considerada como la percepción del bienestar subjetivo, la cual se consigue a partir del logro de las metas y la valoración que le dé el grupo y la sociedad de pertenencia. Sin embargo, es necesario señalar que este término ha sido usado indistintamente como sinónimo de bienestar, felicidad o satisfacción con la vida (Camfield \& Skenvington, 2008; Veenhoven, 2000), estado funcional, valores vitales e incluso salud mental o estado de salud (Meeberg, 1993). Ferrans \& Powers (1985) señalaban que en la década de los ochenta no había un acuerdo común, lo que originaba inconsistencias en las definiciones y en su evaluación. En los noventa, Gill \& Feinstein (1994) revisaron las diferentes formas de medición de la calidad de vida y concluyeron que se estaba evaluando de forma inadecuada, en la medida que no se contaba con una definición clara de este constructo.

En la actualidad, no hay una sola orientación para medir la calidad de vida, pues existen instrumentos genéricos y específicos (Cardona-Arias \& HiguitaGutiérrez, 2014; Urzúa \& Caqueo-Urízar, 2012). Y esto se debe a la multiplicidad de definiciones, ya que aún no se evidencia una forma integradora que considere los aspectos objetivos y subjetivos de este constructo (Durán, Prieto \& García, 2017; Martínez, Vergel \& Zafra, 2015). Sin embargo, la psicología se ha enfocado en la dimensión personal del concepto (Ardila, 2003; Cardona \& Agudelo, 2005). Por su parte, Casas (2004) alude a dos indicadores sociales orientados a la satisfacción general con la vida (Diener, Emons, Larsen \& Griffin, 1985) y la satisfacción con determinados aspectos de la vida (Cummins, 2003). Este último aspecto es sobre el cual se sustenta el análisis instrumental que se presenta en este estudio.

Por su parte, Urzúa \& Caqueo-Urizar (2012) clasificaron las diferentes definiciones y encontraron que se organizaban en cinco categorías: aquellas definiciones que aluden a la calidad de vida en término de condiciones de vida (Hollanflsworth, 1988), satisfacción con la vida
(Michalos, 2004), a la interacción entre los aspectos anteriores -visión objetiva y subjetiva- (Felce \& Perry, 1995), la incorporación de valores a la integración de condiciones y satisfacción con la vida (The WHOQOL Group, 1995) y una quinta clasificación, que enfatiza la evaluación; en donde la Calidad de Vida constituye un constructo latente que se deriva de otros constructos o dominios, tal como lo plantea Campbell (1981).

Como se observa, existen varias formas de definir y evaluar la calidad de vida (Casas, 2004), lo cual constituye una limitación y genera la necesidad de reconceptualizar el constructo a partir de elementos similares en los conceptos propuestos (Benítez, 2016).

En cuanto a los instrumentos que se utilizan en la actualidad, en Colombia, Martínez, et al (2015), construyeron y determinaron las evidencias de validez y fiabilidad de un instrumento para medir la calidad de vida en jóvenes. El análisis factorial arrojó las siguientes áreas: satisfacción con la vida, calidad espacial y percepción del espacio público e índice de pobreza. Otro instrumento que es usado para evaluar la Calidad de Vida es el WHOQOL-BREF, el cual recoge información acerca de las siguientes dimensiones: salud física, psicológica, relaciones sociales y medio ambiente (Cardona-Arias \& Higuita-Gutiérrez, 2014) y ha demostrado tener un buen funcionamiento (Bowden \& Fox-Rushby, 2003).

En México López, González \& Tejada (2016) estudiaron las propiedades psicométricas de la Escala de Calidad de Vida WHOQOL-BREF en adultos que incluyó la participación de estudiantes universitarios. Aunque se mantuvieron las cuatro dimensiones, se eliminaron algunos reactivos.

En Argentina, Jatuff, Zapata-Vega, Montenegro \& Mezzich (2007) validaron el índice Multicultural de Calidad de vida en estudiantes, profesionales de la salud y pacientes psiquiátricos. Los resultados mostraron que además cuenta con altos índices de consistencia interna.

En el Perú, la Escala de Calidad de Vida de Barnes \& Olson (1982), constituye uno de los instrumentos más usados en nuestro medio (Anicama, Mayorga \& Henostroza, 2001; Becerra, 2016; Benavides \& Tarrillo, 2016; Castillo, 2015; Chávez, 2015; García, 2008; Grimaldo, 2011; García, 2018; Tello, 2018; Tueros, 2018). Grimaldo (2009), determinó las características psicométricas de la Escala de Calidad de Vida de Barnes 
\& Olson (1982) a partir de la evaluación de siete áreas específicas o dominios de la vida. Según Michalos (2004), la calidad de vida puede ser evaluada a partir de diferentes dominios de la vida; así las personas evalúan su calidad de vida a partir de expectativas y sentimientos (Campbell, 1981). Por su parte, Olson \& Barnes (1982), señalan que un aspecto común en los trabajos sobre calidad de vida constituye el nivel de satisfacción con las diferentes situaciones vitales o dominios a los que se enfrentan las personas. "Así, la manera como cada persona logra satisfacer estos dominios constituye un juicio individual y subjetivo de la forma como satisface sus necesidades y logra sus intereses en el ambiente" (Grimaldo, 2012, p. 91).

Según Schmidt, Raimundi \& Molina (2015) luego de realizar una revisión encontraron que existen aproximadamente quince dominios vitales relevantes, entre los que se encuentran los siguientes "salud cognitiva, salud económica, salud emocional, salud ambiental, intereses intelectuales, salud comportamental, cuidados de la salud, ocio, satisfacción vital general, actividades no recreativas (incluye trabajo y estudio), salud física, sueño, espiritualidad y sentido, y vitalidad" (p. 309).

Conceptualmente la calidad de vida se define como "un proceso dinámico, complejo y multidimensional; basado en la percepción subjetiva del grado de satisfacción que una persona ha alcanzado en relación a las dimensiones de su ambiente" (Grimaldo, 2011, p. 175). Operacionalmente, se define como el nivel de satisfacción obtenido a partir de los puntajes en los dominios: Hogar y bienestar económico, amigos, vecindario y comunidad, vida familiar y familia extensa, educación y ocio, medios de comunicación, religión y salud, que corresponde a la adaptación realizada por Grimaldo (2009).

El dominio Hogar y bienestar económico, está orientada a recoger información acerca de las condiciones de la vivienda, responsabilidades en la casa, capacidad de la familia para satisfacer las necesidades básicas y lujos y la cantidad de dinero que tiene (Grimaldo, 2012). Este dominio está relacionado con el encontrado por Schmidt, et al. (2015) conceptualizado como dominio salud económica. El dominio amigos, vecindario y comunidad se refiere a la calidad de vida en relación con los amigos, facilidades para hacer compras en el vecindario, seguridad en la comunidad, el barrio donde vive y las facilidades para la recreación(Grimaldo, 2003). De la misma manera, Schmidt, et al. (2015), incluyen ítems relacionados con los amigos.

El dominio vida familiar y familia extensa incluye los ítems relacionados con la familia, hermanos, número de hijos y la relación con los demás parientes (Grimaldo, 2003). Schmidt, et al. (2015), lo representan en el "factor vida familiar, el cual incluye ítems de familia, pareja, e hijos" (p. 309). El dominio educación y ocio recoge información acerca de la actual situación escolar, tiempo libre y la manera como se usa el tiempo libre (Grimaldo, 2003). Así, en el dominio medios de comunicación hace referencia a la cantidad de tiempo que los miembros de la familia pasan viendo televisión, calidad de los programas de televisión, cine, periódicos y revistas (Grimaldo, 2003; Olson \& Barnes, 1982). Finalmente, el dominio religión recoge información acerca de la vida religiosa de la familia y la comunidad y el dominio salud, se orienta a evaluar la propia salud y la de los otros miembros de la familia (Grimaldo, 2003; Olson \& Barnes, 1982).

El propósito del presente estudio fue determinar las evidencias psicométricas de la Escala de Calidad de Vida de Barnes y Olson (ECVOB) -dado que es un instrumento muy usado en el medio, pese a que la versión utilizada data de más de una década (Grimaldo, 2009)- en escolares y estudiantes universitarios. Además, es necesario contar con escalas validadas en nuestro contexto, y por estar dirigido a una población de adolescentes y jóvenes, expuestos a diversos factores de riesgo tales como el consumo de sustancias, conducta antisocial, familias disfuncionales, entre otros problemas que impactan en su desarrollo y que lo convierten en un sector vulnerable (Castro \& Llanes, 2006; Lara, Saldaña, Fernández \& Delgadillo, 2015), generando un estilo de vida poco saludable (González, Salinas, Torre, \& Mora, 2014; Pérez-de-Celis, Lara, Somodevilla, Pineda \& Colmenares; 2016). Incluso se ha encontrado que la Calidad de Vida influye de manera determinante en el rendimiento del estudiante (Durán, et al., 2017).

El objetivo general por tanto fue determinar las evidencias psicométricas de la Escala de Calidad de Vida de Olson y Barnes (ECVOB) en escolares y estudiantes universitarios. Para ello se planteó como objetivos específicos, explorar la estructura interna a través del 
análisis Factorial Confirmatorio y establecer la confiabilidad a través de la consistencia interna en la muestra referida.

\section{MÉTODO}

\section{Diseño y participantes}

El diseño de la presente investigación es instrumental (Ato, López, \& Benavente, 2013), pues se analizan las evidencias de validez y fiabilidad de un instrumento de medida psicológica, y se siguen los estándares de validación desarrollados conjuntamente por la American Educational Research Association (AERA), American Psychological Association (APA) y el National Council on Measurement in Education (NCME) (AERA, APA \& NCME 2018).

La muestra del estudio estuvo compuesta por 1239 estudiantes de Lima Metropolitana, de los cuales 953 fueron estudiantes de dos instituciones educativas de nivel secundario, una pública (64.70\%), otra privada (35.30\%), y 286 estudiantes de cursos generales primer ciclo de una universidad pública de Lima. Todos elegidos intencionalmente debido a su accesibilidad. Las edades de los participantes están comprendidas entre los 14 y 26 años ( $M=22.45$ años; $D E=3.75)$, el $43.30 \%$ del total de la muestra fueron mujeres $(M=21.43$ años; $D E=3.56) \mathrm{y}$ $56.70 \%$ varones $(M=23.46$ años; $D E=3.94)$, de nivel sociocultural y económico medio.

\section{Instrumentos}

Escala de Calidad de Vida de Olson \& Barnes(ECVOB abreviada; Barnes \& Olson, 1982). Se utilizó la versión validada en el contexto peruano adaptada por Grimaldo (2003), compuesta por 25 ítems escalados en formado Likert de cinco puntos (1 = Insatisfecho, $2=$ Un poco satisfecho, 3= Más o menos satisfecho, 4= Bastante satisfecho, 5 = Completamente satisfecho). La ECVOB evalúa los niveles de calidad de vida y tiene siete áreas: Hogar y bienestar económico, amigos, vecindario y comunidad, vida familiar y familia extensa, educación y ocio, medios de comunicación, religión y salud. Los ítems se califican de forma directa, por lo que mayor puntuación hace referencia a mayor presencia del constructo evaluado. Se aplica a personas con edades de 13 años a más. La administración puede ser individual y colectiva, y su aplicación dura en promedio 25 minutos. El estudio de validación peruano desarrollado por Grimaldo (2009) identifico evidencias de validez estructural a partir de un análisis factorial exploratorio, arrojando una medida Kaiser-Meyer-Olkin (KMO) de .87. Así, a partir de la prueba de esfericidad de Barlett se pudo determinar una matriz de correlaciones positiva $(p<.01)$, el criterio de autovalor mayor que 1, explicó el 56\% de la varianza total, esto significa que los factores alcanzaron a explicar la correlación entre ítems. El análisis de fiabilidad se realizó por el método de consistencia interna reportando un coeficiente Alfa de .82 para el Factor Bienestar Económico; .67 para Amigos, Vecindario y Comunidad; .71 para Vida Familiar; .56 para Educación y Ocio; .64 de Medios de Comunicación; .47 para Religión y .58 para el factor Salud.

\section{Procedimiento}

En el proceso de recolección de datos, se solicitó autorización a los directores de las instituciones educativas a quienes se les presentó el proyecto. La evaluación se realizó en las instalaciones de estas instituciones y se siguieron los lineamientos establecidos por AERA, APA y NCME (2018), se informó a los participantes el propósito de la evaluación y se les indicó que podían solicitar sus resultados de forma individual, con total confidencialidad. Así, solo fueron incluidos en el estudio quienes presentaron el consentimiento informado de los padres y/o apoderados, y aceptaron participar a través del llenado del asentimiento informado. Respecto a las consideraciones éticas, en todo momento se siguieron las normas establecidas en el capítulo III, Investigación del Código de Ética y Deontología del Colegio de Psicólogos del Perú (2017), con lo cual se garantizó el anonimato y salvaguarda de los participantes.

\section{Análisis de datos}

El análisis estadístico se realizó mediante etapas. En la primera etapa se analizaron las medidas descriptivas de los ítems y sus características distribucionales. El supuesto de normalidad se evalúo a través de los coeficientes de asimetría y curtosis considerando como criterio los valores dentro del rango de \pm 1.5 (Pérez \& Medrano, 2010). En la segunda etapa, para identificar las evidencias de validez estructural se realizó un Análisis Factorial Confirmatorio (AFC) para dos modelos: el modelo original (Olson \& Barnes, 1982; Grimaldo, 2003) 
compuesto por siete factores: Hogar y Bienestar Económico (1, 2, 3, 4, 5), Amigos, Vecindario y Comunidad $(6,7,8,9,1)$, Vida Familiar y Familia Extensa $(11,12,13$, 14), Educación y Ocio $(15,16,17)$, Medios de Comunicación (18, 19, 2, 21), Religión (22, 23), Salud (24, 25). El segundo modelo compuesto por seis factores (F1: 1, 2, 15; F2: 19, 20, 21; F3: 16, 17, 18, 22; F4: 6, 7, 9, 10, 11 , 12, 13, 14; F5: 3, 4, 5, 8; F6: 23, 24, 25). Además, se consideraron pesos factoriales aceptables a partir de .40 (Williams, Onsman, \& Brown, 2010).

El método de estimación empleado fue Weighted Least Square Mean and Variance Adjusted (WLSMV), debido a la naturaleza categórica de las variables de estudio (Domínguez-Lara, 2014; Verdam Oort, \& Sprangers, 2016), además de ser un estimador más confiable en muestras pequeñas ( $\mathrm{Li}, 2014)$. Finalmente, se compararon los índices de ajuste de ambos modelos, entre los que destacaban la razón chi cuadrado entre los grados de libertad ( $\chi 2 / g$ ) con valores esperados menores a 3, Root Mean Square Error of Approximation (RMSEA) y Standarized Root Mean Square Residual (SRMR) en ambos casos se esperaron valores por debajo a .08 sugeridos por Bentler \& Bonnet (1980). Se incluyó el Comparative Fit Index (CFI) de bondad de ajuste de Jöreskog \& Sörbom (1986) para verificar el ajuste incremental, cuyos valores aceptables se encontraban por encima de .95, al igual que el Índice de Tucker-Lewis (TLI) el cual permitió cotejar el ajuste de parsimonia (Kline, 2016).

Adicionalmente, a partir de las cargas factoriales, se calculó la varianza promedio extraída (Average Variance Extracted [AVE]) considerando valores alrededor del .50 como satisfactorios, con ello se verificaron las evidencias de validez interna convergente (Fornell \& Larcker, 1981; Navarro, Dominguez, \& Medrano, 2019).

Las evidencias de fiabilidad por consistencia interna se evaluaron a través del coeficiente omega $(\omega)$, debido a que este coeficiente sigue siendo imparcial con elementos congéneres con errores no correlacionados. Adicionalmente, se calculó el coeficiente de fiabilidad compuesta (Compositive Reliability [CR]).

\section{RESULTADOS}

\section{Análisis descriptivo}

En la Tabla 1, se presenta el análisis descriptivo de los ítems que componen el ECVOB. Estos valores se calcularon a partir de las puntuaciones obtenidas en cada uno de los ítems que conforman el instrumento. Evidenciando que los ítems $17(M=3.73, D E=1.08)$ y 22 $(M=3.81, D E=1.00)$ presentan las medias aritméticas más altas, mientras que la media más baja está presente en los ítems $14(M=3.06, D E=1.05)$ y $15(M=3.06, D E=$ $1.18)$. En cuanto a la variabilidad, se aprecia que los ítems $1(M=3.10, D E=1.32)$ y $3(M=3.20, D E=1.39)$ son los que presentan mayor dispersión. La asimetría y curtosis fluctuaron entre valores inferiores $a \pm 1.5$, indicando que la distribución de los ítems se aproxima a una distribución univariante normal (Pérez \& Medrano, 2010)

Tabla 1. Estadísticos descriptivos

\begin{tabular}{|c|c|c|c|c|}
\hline Ítems & $M$ & $D E$ & $g_{1}$ & $g_{2}$ \\
\hline $\begin{array}{l}\text { CV1. Tus actuales condiciones de } \\
\text { vivienda }\end{array}$ & 3.10 & 1.32 & -0.12 & -1.10 \\
\hline $\begin{array}{l}\text { CV2. Tus responsabilidades en la } \\
\text { casa }\end{array}$ & 3.34 & 1.15 & -0.33 & -0.62 \\
\hline $\begin{array}{l}\text { CV3. La capacidad de tu familia para } \\
\text { satisfacer sus necesidades básicas }\end{array}$ & 3.20 & 1.39 & -0.22 & -1.18 \\
\hline $\begin{array}{l}\text { CV4. La capacidad de tu familia para } \\
\text { darles lujos }\end{array}$ & 3.57 & 1.11 & -0.55 & -0.29 \\
\hline $\begin{array}{l}\text { CV5. La cantidad de dinero que } \\
\text { tienes para gastar }\end{array}$ & 3.16 & 1.11 & -0.24 & -0.54 \\
\hline CV6. Tus amigos & 3.44 & 1.06 & -0.36 & -0.50 \\
\hline $\begin{array}{l}\text { CV7. Las facilidades para hacer } \\
\text { compras en tu comunidad }\end{array}$ & 3.42 & 1.11 & -0.48 & -0.34 \\
\hline CV8. La seguridad en tu comunidad & 3.41 & 1.13 & -0.43 & -0.45 \\
\hline CV9. El barrio donde vives & 3.42 & 1.06 & -0.41 & -0.31 \\
\hline $\begin{array}{l}\text { CV10. Las facilidades para recreación } \\
\text { (parque, campos de juegos, etc.) }\end{array}$ & 3.54 & 1.06 & -0.48 & -0.16 \\
\hline CV11. Tu familia & 3.43 & 1.06 & -0.40 & -0.34 \\
\hline CV12. Tus hermanos & 3.50 & 0.97 & -0.40 & -0.10 \\
\hline $\begin{array}{l}\text { CV13. El número de hijos en tu } \\
\text { familia }\end{array}$ & 3.67 & 1.00 & -0.50 & -0.17 \\
\hline $\begin{array}{l}\text { CV14. Tu relación con tus parientes } \\
\text { (abuelos, tíos, primos) }\end{array}$ & 3.06 & 1.05 & -0.13 & -0.43 \\
\hline CV15. Tu relación con tus parientes & 3.06 & 1.18 & -0.17 & -0.76 \\
\hline CV16. Tu actual situación escolar & 3.60 & 1.19 & -0.54 & -0.61 \\
\hline CV17. El tiempo libre que tienes & 3.73 & 1.08 & -0.72 & -0.01 \\
\hline $\begin{array}{l}\text { CV18. La forma como usas tu tiempo } \\
\text { libre }\end{array}$ & 3.44 & 1.01 & -0.36 & -0.18 \\
\hline
\end{tabular}




\begin{tabular}{lcccc} 
CV19. La cantidad de tiempo que los & 3.54 & 1.06 & -0.41 & -0.32 \\
miembros de tu familia pasan viendo & & & & \\
TV & & & & \\
$\begin{array}{l}\text { CV2. Calidad de los programas de } \\
\text { televisión }\end{array}$ & 3.37 & 1.07 & -0.33 & -0.39 \\
$\begin{array}{l}\text { CV21. Calidad del cine } \\
\text { CV22. La calidad de periódicos y }\end{array}$ & 3.21 & 1.06 & -0.25 & -0.41 \\
revistas & & 1.00 & -0.67 & 0.03 \\
$\begin{array}{l}\text { CV23. La vida religiosa detu familia } \\
\text { CV24. La vida religiosa detu }\end{array}$ & 3.37 & 1.10 & -0.37 & -0.44 \\
comunidad & 3.59 & 1.08 & -0.60 & -0.18 \\
CV25. Tu propia salud & & & & \\
\hline
\end{tabular}

Nota: M=Media; DE=Desviación Estándar; $g_{1}=$ Asimetría; $g_{2}=$ Curtosis

\section{Evidencias de validez basada en la estructura intema}

Para evaluar la validez estructural de la ECVOB se realizó un Análisis Factorial Confirmatorio con un estimador robusto (WLSMV), por la naturaleza categórica de las variables (Brown, 2015) y el tamaño de muestra (Li, 2014). De esta forma, se evaluó el ajuste de los dos modelos de ECVOB. En la Tabla 2, respecto a la estructura interna del ECVOB del modelo original (Modelo 1) de siete factores, se aprecia que alcanza cargas factoriales satisfactorias $(\Lambda>.40)$ en cada uno de sus factores (Williams, Onsman, \& Brown, 2010). Se calculó la varianza promedio extraída (Average Variance Extracted [AVE]) cuyos valores se encuentran por debajo de lo esperado (.50), lo que indica que la estructura original no presenta validez interna convergente (Fornell \& Larcker, 1981; Navarro-Loli, Domínguez-Lara, \& Medrano, 2019). En general, se aprecia que la mayoría de las cargas factoriales de cada factor son inferiores a las covarianzas con otros factores. Así, el F1 presenta covarianzas que fluctúan entre .21 (F6) y .79 (F2); F2 alcanza covarianzas que van .57 (F6) a .85 (F3); F3 cuyas covarianzas oscilan entre .60 (F5) y .76 (F4); F4 con covarianzas que van de .73 (F7) a .97 (F6); F5 reporta covarianza entre .66 (F6) y .67 (F7) alcanza una covarianza de .89 (F6). Obteniendo una matriz de correlaciones positiva y con coeficientes que fluctúan entre .55 y .14. Se observa, que la covarianza entre el F1 y F2 es igual a .79.

En cuanto al Modelo 2, el cual se desprende de una recomposición de los ítems a partir de su carga factorial, de los índices de bondad de ajuste y los coeficientes de verificación (AVE, CR y AlIC). Así, las saturaciones de los factores del Modelo 2, evidencia cargas factoriales más homogéneas con respecto al Modelo 1. Asimismo, las covarianzas del Factor 1, fluctúan entre .30 (F6) y .90 (F3); para el Factor 2 las covarianzas van .52 (F3) a .69 (F5); en el Factor 3 las covarianzas oscilan entre .15 (F5) y .77 (F6); el Factor 4 presenta covarianzas que van .67 (F5) a .68 (F6); el Factor 5 tiene una covarianza .43 (F6), estas covarianzas en su mayoría son superiores a las cargas factoriales. A diferencia del Modelo 1, los valores del AVE son sustancialmente superiores; sin embargo, alcanzando valores cercanos a .50 (Fornell \& Larcker, 1981; Navarro, et al., 2019). Las correlaciones entre los factores son positivas, pero son inferiores a las covarianzas entre factores.

Tabla 2. Análisis factorial confirmatorio y fiabilidad de ECVOB

\begin{tabular}{|c|c|c|c|c|c|c|c|c|c|c|c|c|c|c|c|c|}
\hline \multicolumn{10}{|c|}{ Modelo 1 (7 factores) } & \multicolumn{7}{|c|}{ Modelo 2 (6 factores) } \\
\hline Ítems & $\mathrm{F} 1$ & $\mathrm{~F} 2$ & F3 & F4 & F5 & F6 & F7 & $\Lambda^{2}$ & Ítems & $\mathrm{F} 1$ & $\mathrm{~F} 2$ & F3 & $\mathrm{F} 4$ & F5 & F6 & $\Lambda^{2}$ \\
\hline CV1 & .81 & & & & & & & .66 & CV1 & .84 & & & & & & .71 \\
\hline CV2 & .77 & & & & & & & .59 & $\mathrm{CV} 2$ & .79 & & & & & & .63 \\
\hline CV3 & .68 & & & & & & & .47 & CV15 & .65 & & & & & & .42 \\
\hline CV4 & .64 & & & & & & & .41 & $\mathrm{CV} 2$ & & .71 & & & & & .5 \\
\hline CV5 & .62 & & & & & & & .38 & CV19 & & .78 & & & & & .6 \\
\hline CV6 & & .63 & & & & & & .39 & CV21 & & .59 & & & & & .35 \\
\hline CV7 & & .46 & & & & & & .21 & CV16 & & & .60 & & & & .36 \\
\hline CV8 & & .56 & & & & & & .31 & CV17 & & & .72 & & & & .52 \\
\hline CV9 & & .57 & & & & & & .33 & CV18 & & & .58 & & & & .34 \\
\hline CV11 & & & .56 & & & & & .31 & CV14 & & & & .52 & & & .27 \\
\hline CV12 & & & .62 & & & & & .39 & CV13 & & & & .53 & & & .28 \\
\hline CV13 & & & .58 & & & & & .33 & CV6 & & & & .66 & & & .42 \\
\hline
\end{tabular}




\begin{tabular}{|c|c|c|c|c|c|c|c|c|c|c|c|c|c|c|c|c|}
\hline CV14 & & & .56 & & & & & .32 & CV11 & & & & .51 & & & .26 \\
\hline CV15 & & & & .58 & & & & .33 & $\mathrm{CV} 12$ & & & & .58 & & & .33 \\
\hline CV16 & & & & .45 & & & & .21 & CV9 & & & & .59 & & & .35 \\
\hline CV17 & & & & .60 & & & & .36 & CV1 & & & & .44 & & & .20 \\
\hline CV18 & & & & & .45 & & & .2 & CV7 & & & & .48 & & & .23 \\
\hline CV19 & & & & & .76 & & & .57 & CV4 & & & & & .64 & & .41 \\
\hline CV2 & & & & & .69 & & & .48 & CV3 & & & & & .69 & & .47 \\
\hline CV21 & & & & & .58 & & & .33 & CV5 & & & & & .61 & & .37 \\
\hline CV22 & & & & & & .64 & & .42 & CV8 & & & & & .63 & & .39 \\
\hline $\mathrm{CV}_{23}$ & & & & & & .66 & & .44 & CV24 & & & & & & .64 & .41 \\
\hline CV24 & & & & & & & .65 & .42 & CV23 & & & & & & .66 & .44 \\
\hline CV25 & & & & & & & .67 & .45 & CV25 & & & & & & .66 & .43 \\
\hline & & & & & Cova & רzas & tercc & acion & Factoria & Mode & y Mo & & & & & \\
\hline & 1 & 2 & 3 & 4 & 5 & 6 & 7 & & & 1 & 2 & 3 & 4 & 5 & 6 & \\
\hline F1 & & .55 & .34 & .21 & .40 & .14 & .24 & - & F1 & & .41 & .50 & .47 & .59 & .20 & - \\
\hline $\mathrm{F} 2$ & .79 & & .53 & .36 & .52 & .35 & .39 & - & $\mathrm{F} 2$ & .57 & & .36 & .47 & .48 & .42 & - \\
\hline F3 & .48 & .85 & & .46 & .41 & .38 & .38 & - & F3 & .09 & .52 & & .40 & .70 & .51 & - \\
\hline F4 & .44 & .63 & .76 & & .45 & .51 & .42 & - & F4 & .63 & .67 & .58 & & .47 & .45 & - \\
\hline F5 & .56 & .79 & .60 & .71 & & .44 & .43 & - & F5 & .81 & .69 & .15 & .68 & & .28 & - \\
\hline F6 & .21 & .57 & .64 & .97 & .67 & & .50 & - & F6 & .30 & .60 & .77 & .67 & .43 & & - \\
\hline F7 & .38 & .65 & .65 & .73 & .66 & .89 & & - & - & - & - & - & - & - & - & - \\
\hline \multicolumn{17}{|c|}{ Medidas de fiabilidad } \\
\hline$\omega$ & .79 & .63 & .63 & .56 & .67 & .54 & .56 & - & $\omega$ & .77 & .69 & .70 & .73 & .69 & .66 & - \\
\hline$A / / C$ & .42 & .25 & .29 & .20 & .32 & .37 & .39 & - & $A / / C$ & .51 & .41 & .36 & .25 & .36 & .39 & - \\
\hline AVE & .50 & .29 & .34 & .30 & .40 & .42 & .44 & - & AVE & .58 & .49 & .42 & .30 & .41 & .43 & - \\
\hline$C R$ & .83 & .66 & .67 & .57 & .72 & .59 & .61 & - & $C R$ & .81 & .74 & .74 & .77 & .74 & .69 & - \\
\hline
\end{tabular}

Nota. $\Lambda^{2}=$ Lamda al cuadrado; Bajo la diagonal: covarianza entre factores; Sobre la diagonal: Correlaciones interfactoriales; AllC=Avarege Interitem

Correlation (Correlación Interítem Promedio); AVE=Average Variance Extracted; CR=Composite Reliability

En el primer modelo, donde se conservan los ítems en su versión original, se halló que los índices CFF.93, $T L$ =.91, RMSEA=.085 no ajustan apropiadamente (Ruiz, Pardo \& San Martín, 2010). Mientras que, para el segundo modelo con la reagrupación de los ítems los índices de ajuste alcanzan valores satisfactorios $X^{2}=1473.94$, RMSEA =.065 CFI=.96, TLI=.96 (Keith, 2015; Schumacker \& Lomax, 2016) (ver Tabla 3).

Tabla 3. Índices de bondad de ajuste de dos modelos del AFC de ECVOB

\begin{tabular}{|c|c|c|c|c|c|c|c|c|}
\hline \multirow{2}{*}{ Modelos } & \multirow{2}{*}{$x^{2}$} & \multirow{2}{*}{ g.l. } & \multirow{2}{*}{$p$} & \multirow{2}{*}{$C F I$} & \multirow{2}{*}{$T L I$} & \multirow{2}{*}{ RMSEA } & \multicolumn{2}{|c|}{ IC95\% } \\
\hline & & & & & & & Li & $\angle S$ \\
\hline Modelo 1 & 2758.00 & 252 & $<.01$ & .93 & .91 & .085 & .082 & .091 \\
\hline Modelo 2 & 1473.94 & 237 & $<.01$ & .96 & .96 & .065 & .062 & .068 \\
\hline
\end{tabular}

Nota. $X^{2}=$ Chi cuadrado; g.l.=Grados de Libertad; CF/f́ndice de bondad de ajuste comparativo; TLIfíndice de Tucker Lewis; RMSEA=Raíz del residuo cuadrático promedio de aproximación

En la Figura 1, se presenta el diagrama de senderos de la ECVOB en universitarios limeños, se puede apreciar una estructura de seis factores cuyas cargas factoriales se ubican por encima de .40 los cuales corresponden a valores adecuados (Williams, Onsman, \& Brown, 2010). 


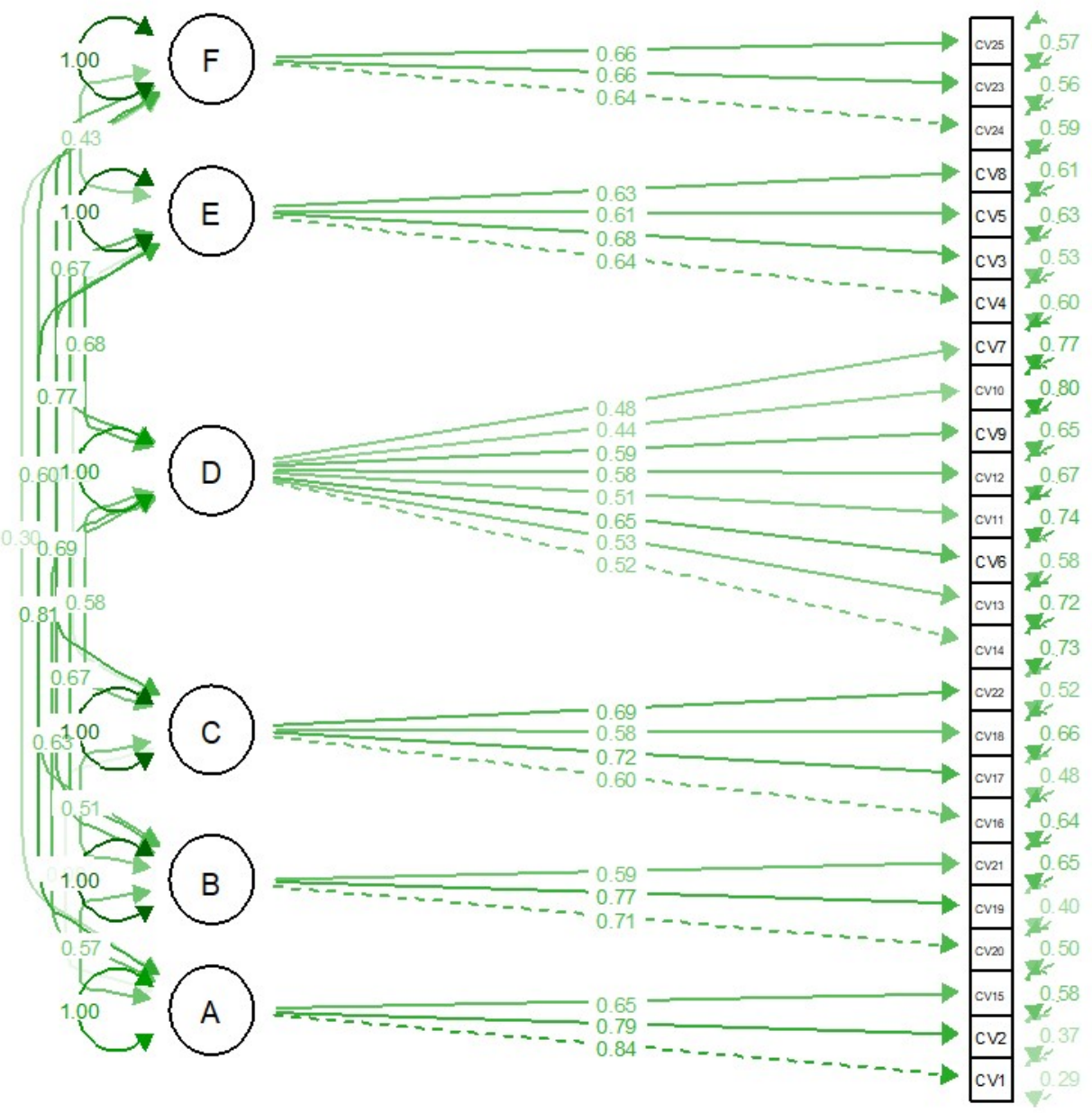

Figura 1. Análisis Factorial Confirmatorio para el Modelo 2

\section{DISCUSIÓN}

El objetivo del presente estudio fue evaluar las propiedades psicométricas referidas a las evidencias de validez de la estructura interna y la fiabilidad de la ECVOB universitarios limeños.

Los resultados muestran una estructura factorial de seis factores la cual difiere parcialmente de la propuesta original de siete factores de Olson \& Barnes (1982); sin embargo, esta reestructuración coincide con otros estudios instrumentales realizados en muestras de adolescentes y universitarios, los cuales sugieren modificaciones en el instrumento (Grimaldo, 2003; 2011; 2012; Becerra, 2016; Tello, 2018, \& Tueros, 2018).

No obstante, en la presente investigación se propone un reagrupamiento de los ítems y la eliminación de uno de estos factores. En el Modelo de seis factores la mayoría de los ítems se mantiene en su dimensión de origen, ordenándose de la siguiente manera el Factor 1
(Hogar), Factor 2 (Medios de comunicación), Factor 3 (Educación y ocio), Factor 4 (Amigos, vecindario, comunidad, vida familiar y extensa), Factor 5 (Economía) y Factor 6 (Salud), alcanzando cargas factoriales satisfactorias (Williams, Onsman, \& Brown, 2010). Desaparece el factor de religión cuyos ítems 22 y 23 se reagrupan en los factores educación y salud respectivamente. Tal como se observa, este modelo propuesto, es congruente con la teoría subyacente del constructo calidad de vida.

La nueva estructura respeta los lineamientos que sugieren un mínimo de tres ítems por factor y que estos alcancen pesos factoriales e índices de ajuste, incrementales y parsimoniosos adecuados (Fabrigar et al., 1999; Ferrando \& Anguiano-Carrasco, 2010 en LloretSegura, et.al, 2014). Estas modificaciones la versión final de la ECVOB (seis factores) es una medida parsimoniosa, robusta e interpretable. 
En lo referente a las evidencias de validez interna, los hallazgos corroboran atributos discriminantes entre los factores y la convergencia de los elementos con el constructo subyacente del cual provienen teóricamente, identificándose que los valores del AVE son sustancialmente superiores en el Modelo 2 (seis factores), dejando evidencia parcial de la diferenciación de los constructos que componen la ECVOB porque los valores del AVE son parcialmente mayores que la covarianza entre factores, y superior que las correlaciones de las puntuaciones entre los factores. Ello a pesar de que solo el primer y segundo factor alcanzan valore AVE adecuados (Fornell \& Larcker, 1981).

Por otra parte, con respecto a las evidencias de fiabilidad, se estimaron a través del Coeficiente Omega (w), debido a la naturaleza ordinal de los ítems, y al incumplimiento del supuesto de tau equivalencia (Cho, 2016; Frías-Navarro, 2019), los valores del coeficiente omega alcanzaron niveles aceptables los cuales fluctúan entre .66 y .77 (Cho \& Kim, 2015, Domínguez-Lara, 2016). Además, se calculó el coeficiente de fiabilidad compuesta el cual obtiene valores por encima de .70 sus dimensiones, a excepción del sexto factor el cual obtiene una fiabilidad compuesta igual a .69 la cual se encuentra dentro de los márgenes tolerables (Zapata \& Canet, 2008). Asimismo, en términos de García (2005) y Katz (2006) los valores hallados para los factores F2 (medios de comunicación), F5 (economía) y F6 (salud) son aceptables. En síntesis, las mediciones de la escala son consistentes, es decir los ítems que componen el instrumento miden un mismo constructo (Medrano \& Pérez, 2018; Shook, Ketchen, Hult, \& Kacmar, 2004). Además, estos resultados coinciden con los reportado en otros estudios (Grimaldo, 2003, 2009).

\section{Conclusiones}

La evidencia empírica demuestra que el constructo estudiado, calidad de vida, está representado en una estructura interna de seis factores presentes en este instrumento, reafirmando su concepto como un proceso dinámico, complejo y multidimensional (Grimaldo, 2003; 2011; 2012; Becerra, 2016; Tello, 2018, \& Tueros, 2018) que considera a sus factores latentes y a sus respectivas variables (ítems).

El estudio ha considerado una muestra cuyas características no escapan a los factores de riesgo de procedencia de instituciones públicas. Es en ese contexto, surge la limitación parcial del empleo del instrumento teniendo en cuenta solo situaciones similares, por lo que los resultados no pueden ser generalizados. Se sugiere para futuras investigaciones optar por muestras de otras características no incluidas en la presente investigación.

Por último, se concluye que los resultados hallados indican que la ECVOB aplicado en estudiantes de secundaria y universitarios peruanos cuenta con adecuadas propiedades psicométricas que permiten considerarlo como una medida válida y confiable que puede ser empleada en investigaciones futuras como instrumento de medición de la calidad de vida.

En cuanto a las limitaciones del estudio, se determinó la muestra de forma no probabilística, por lo cual no se pueden generalizar los resultados. De la misma manera, se incluyeron solo a estudiantes de nivel socio económico medio y que vivían en Lima en el momento del estudio, dejando de lado a estudiantes de otros niveles educativos y de diferentes culturas. Además de considerar muestras de adultos y adultos mayores. En cuanto a los aportes a esta a línea de investigación se sugiere realizar futuros estudios que consideren el análisis de la fiabilidad mediante el test-retest; así como las evidencias de validez convergente y divergente.

\section{Conflicto de intereses}

Los autores declaran no tener ningún conflicto de interés con respecto a la investigación, autoría o publicación de este artículo. La investigación ha sido financiada con recursos propios, sin contar con el subsidio de ninguna institución pública o privada.

\section{REFERENCIAS}

\footnotetext{
American Educational Research Association, American Psychological Association \& National Council On Measurement In Education (2018), Standards for Educational and Psychological Testing, Washington, DC.: American Educational Research Association.
}

Anicama, J., Mayorga, E., \& Henostroza, C. (2001). Calidad de Vida y recaídas en adictos a sustancias psicoactivas de Lima metropolitana. Psicoactiva, 19, 7-27.

Ardila, R. (2003). Calidad de vida: una definición integradora. Revista Latinoamericana de Psicología, 35(2), 161-164. 
Ato, M., López, J., \& Benavente, A. (2013). Un sistema de clasificación de los diseños de investigación en psicología. Revista Anales dt Psicología, 29(3), 138-151. doi:10.6018/analesps.29.3.178511

Barnes, H. L., \& Olson, D. L. (1982). Quality of Life. In D. H. Olson et al. (Eds.), Family inventories: Inventories used in a national survey of families across the family life cycle (pp. 114-125). St Paul: Family Social Science, University of Minnesota.

Becerra, F. (2016). Propiedades Psicométricas de la Escala de Calidao de Vida en estudiantes de Secundaria del Distrito La Peca Bagua. (Tesis para optar el título de Licenciado en Psicología). Universidad César Vallejo, Trujillo, Perú.

Benavides, H., \& Tarrillo, J. (2016). Calidad de Vida y autoestima en gestantes. Centro de Salud José Olaya de Chiclayo. Julio-Agosto, 2016. (Tesis para optar el título de Psicólogo). Universidad Privada Juan Mejía Baca, Chiclayo, Perú.

Benítez, I. (2016). La evaluación de la Calidad de vida: retos metodológicos presentes y futuros, Papeles del Psicólogo, 371), 69-73.

Bentler, P., \& Bonett, D. (1980). Significance tests and goodness of fit in the analysis of covariance structures. Psychological Bulletin, 88, 588-606. doi:10.1037/0033-2909.88.3.588

Bowden A., \& Fox-Rushby J. (2003). A systematic and critical review of the process of translation and adaptation of generic healthrelated quality of life measures in Africa, Asia, Eastern Europe, the Middle East, South America. Social Science \& Medicine, 57, 1289-136. doi:10.1016/S0277-9536(02)00503-8

Brown, T. A. (2015). Confirmatory factor analysis for applied research (2a ed.). New York, NY: Guilford Press.

Camfield, L., \& Skenvington, S. (2008). Ob Subjective well-being and quality of life. Journal of Health Psychology, 13(6), 764-775. doi:10.1177/1359105308093860

Campbell, A. (1981). The sense of well-being in America. McGraw-Hill, New York.

Cardona, A. D., \& Agudelo, G. H. (2005). Construcción cultural del concepto calidad de vida. Revista de la Facultad Nacional dt Salud Pública, 23, 79-9.

Cardona-Arias, J., \& Higuita-Gutiérrez, L.F. (2014). Aplicaciones de un instrumento diseñado por la OMS para la evaluación de la calidad de vida. Revista Cubana de salud Pública, 4(2), 175-189.

Casas, F. (2004). De afrontar problemas sociales a promover la calidad de vida. Revista de Historia de la Psicología, 25(4), 305-322.

Castillo, L. (2015). Satisfacción familiar y calidad de vida en estudiantes de una institución educativa Chiclayo. (Tesis de licenciatura). Universidad Señor de Sipán, Chiclayo, Perú.

Castro M.E., \& Llanes J. (2006). El coeficiente de riesgo psicosocial como medida compleja para el monitoreo y seguimiento de la vulnerabilidad psicosocial de poblaciones estudiantiles. Liberaddictus Cuadernos de Prevención. 91, 84-92.

Chávez, C.P. (2015). Calidad de vida y bienestar psicológico en personas con Discapacidad motriz de un centro de integración de Discapacitados de Trujillo. (Tesis de Licenciatura). Universidad Privada Antenor, Trujillo, Perú.

Colegio de Psicólogos del Perú (2017). Código de Ética y Deontología. Lima: autor.

Cummins, R.A. (2003). Normative life satisfaction: Measurement issues and a homeostatic model. Social Indicators Research, 64, 225256. doi:10.1023/A:1024704320683

Diener, E. (2006). Guidelines for national indicators of subjective wellbeing and ill-being. Journal of Happiness Studies, 74), $397-$ 44.doi:10.1007/s10902-006-9000-y

Diener, E. D., Emmons, R. A., Larsen, R. J., \& Griffin, S. (1985). The satisfaction with life scale. Journal of Personality Assessment, 4911), 71-75. doi:10.1207/s15327752jpa4901_13

Domínguez-Lara, S. (2014). ¿Matrices policóricas/tetracóricas o matrices Pearson? Un estudio metodológico. Revista Argentina de Ciencias del Comportamiento (RACC) 6 (1), 39-48. doi:10.21500/19002386.2134

Domínguez-Lara, S. (2016). Evaluación de la confiabilidad del constructo mediante el Coeficiente $\mathrm{H}$ : breve revisión conceptual y aplicaciones, Psychologia: Avances en la disciplina, 102),.87-94. doi:10.21500/19002386.2134

Durán, S., Prieto, R., \& García, J. (2017). Influencia de la Calidad de Vida en el rendimiento del estudiante universitario. En J. Hernández, J. Barboza \& I. Muñoz. (Eds), Calidad de Vida, Inclusión y Bienestar Humano (pp. 89-11), Sucre: CECAR-UNERMB. doi:1.21892/97898427215

Fabrigar, L. R., Wegener, D., Maccallum, R., \& Strahan, E. (1999) Evaluating the use of exploratory factor analysis in psychological research, Psychological Methods, 4(1), 272-299. doi:10.1037/1082-989X.4.3.272

Felce, D., \& Perry, J. (1995). Quality of life: Its definition and measurement. Research in developmental disabilities, 16, 51 74. doi:10.1016/0891-4222(94)00028-8

Ferrando, P. J., \& Anguiano-Carrasco, C. (2010), El análisis factorial como técnica de investigación en Psicología, Papeles del Psicólogo, 31(1), 18-33.

Ferrans, C.E. \& Powers, M.J. (1985). Quality of life index development and psychometric properties. Advances in Nursing Science, 8(1), 15-24. doi:10.1097/00012272-198510000-00005

Fornell, C., \& Larcker, D. F. (1981). Evaluating structural equation models with unobservable variables and measurement error. Journal of Marketing Research, 18, 39-50. doi:10.1177/002224378101800104 
García, C. (2005), "Estadística con SPSS y metodología de la investigación", en René Landero, y Mónica González, Lamedición en las ciencias sociales y en la psicología. México, DF:Trillas

García, C. R. (2008). Niveles de Calidad de Vida en Farmacodependientes de tres centros de rehabilitación terapéutica según tiempo de internamiento de la ciudad dt Lima. (Tesis para optar el Título de Licenciada en Psicología), Universidad de San Martín de Porres, Lima, Perú.

García, C. R. (2018). Calidad de Vida y estilos de crianza en estudiantes universitarios de Lima Metropolitana. (Tesis para optar grado d $\epsilon$ Maestra en Psicología Educativa). Universidad de San Martín de Porres, Lima, Perú.

Grimaldo, M. (2003). Escala de Calidad de Vida de Olson \& Barnes. Lima: Universidad de San Martín de Porres.

Grimaldo, M. (Julio de 2009). Calidad de Vida y Afectos en escolares de Lima. En XXXII Congreso Interamericano de Psicología, $29 \mathrm{~d} \epsilon$ julio-2 de agosto 2009 (pág. 445). Guatemala: Sociedad Interamericana de Psicología.

Grimaldo, M. (2011). Calidad de vida en profesionales de la salud en la ciudad de Lima, Liberabit, 172), 173-185.

Grimaldo, M. (2012), Calidad de vida en estudiantes de secundaria de la ciudad, Avances en Psicología, 2(1), 89-11. doi:10.33539/avpsicol.2012.v20n1.1946

Gill, T. M., \& Feinstein, A.R. (1994). A critical Appraisal of the quality of life measurements (abstract). Journal of the American Medicaı Association, 272, 619-626. doi:10.1001/jama.1994.03520080061045

González, D., Salinas, A., Torre P., \& Mora F. (2014). Necesidades de salud sexual y reproductiva en estudiantes de una universidad pública mexicana. Global Health Promotion, 21(3), 69-79. doi:10.1177/1757975913516653

Hollanflsworth J. G. Jr. (1988) Evaluating the impact of medical treatment on the quality of life: A 5-year update. Social Science and Medicine, 26, 425-434. doi:10.1016/0277-9536(88)90311-5

Jatuff, D., Zapata-Vega, M.I., Montenegro, R., \& Mezzich, J.E. (2007). El índice Multicultural de calidad de Vida en Argentina: un estudio de validación. Actas Españolas de Psiquiatría, 35(4), 253-258.

Jöreskog, K. G., \& Sörbom, D. (1986). Lisrel VI: Analysis of Linear Structural Relationships by Maximum Likelihood and Leas Square Methods. Mooresville, IN: Scientific Software, Inc.

Katz, M. H. (2006). Multivariable analysis (2aed.), Cambridge: Cambridge University Press.

Keith, T. Z. (2015). Multiple Regression and Beyond: An Introduction to Multiple Regression and Structural Equation Modeling. New York: Routledge. doi:10.4324/9781315749099
Kline, R. (2016). Principles and Practice of Structural Equation Modeling (4th Edition). New York: The Guilford Press

Lara, N., Saldaña, Fernández, N., \& Delgadillo, H.J. (2015). Salud, Calidad de vida y entorno universitario en estudiantes mexicanos de una Universidad pública. Hacia la promoción de la salud. 2022), 102117. doi:10.17151/hpsal.2015.20.2.8

$\mathrm{Li}, \mathrm{C} . \mathrm{H}$. (2014). The performance of MLR, USLMV, and WLSMV estimation in structural regression models with ordinal variables (Disertación de Doctorado, Michigan State University, East Lansing, MI, Estados Unidos).

López, J., González, R., \& Tejada, J. (2016). Propiedades psicométricas de la versión en español de la escala decalidad de vida WHO QoL BREF en una muestra de adultos mexicanos, Revista Iberoamericana de Diagnóstico y evaluación, 44(2), 105-115. doi:10.21865/RIDEP44.2.09

Lloret-Segura, S., Ferreres-Traver, A., Hernández-Baeza, A., \& TomásMarco, I. (214). El análisis factorial exploratorio de los ítems: una guía práctica, revisada y actualizada. Anales de Psicología, 3(3), 1151-1169. doi:1.618/analesps.3.3.199361

Martínez, J. J., Vergel, M., \& Zafra (2015). Validez de instrumento para medir la calidad de vida en la juventud: vihda, Revista Logos, Ciencia \& Tecnología, 71 1), 17-26. doi:10.22335/rlct.v7i1.206

Medrano, L., \& Pérez, E. (2018). Manual de Psicometría y evaluación Psicológica. Córdoba: Editorial Brujas

Meeberg, G. A. (1993). Quality of life: a concept analysis. Journal of Advanced Nursing, 18(1), 32-38. doi:10.1046/j.1365-2648.1993.18010032.x

Michalos, A. (2004). Social Indicators research and health related quality of life research. Social Indicators Research, 65, 27-72. doi:10.1023/A:1025592219390

Navarro-Loli, J., Dominguez-Lara, S., \& Medrano, L. (2019) Estructura interna del Cognitive emotion regularion questionnaire (CERQ18) en una muestra de adolescentes peruanos. Revista Iberoamericana de Diagnóstico y Evaluación. 54(1), 165-178. doi:10.21865/RIDEP54.1.13

Barnes, H. L., \& Olson, D. L. (1982). Quality of Life. In DH Olson et al. (Eds), Family inventories used in a national survey of families across the family life cycle (pp. 114-125). St. Paul: Family Social Sciencie, Universidad of Minnesota.

Organización Mundial de la Salud. (1998). Promoción de la Salud. Glosario. Ginebra; autor. Recuperado de http://www.bvs.org.ar/pdf/glosario sp.pdf

Pérez, E., \& Medrano, L. (2010). Análisis Factorial Exploratorio: Bases Conceptuales y Metodológicas. Revista Argentina de Ciencias de Comportamiento, 2(1), 58- 66. 
Pérez-de-Celis Herrero, C., Lara, C., Somodevilla, M.J., Pineda, I.H., \& Colmenares, E. (2016). Estilo de vida de los estudiantes $d \epsilon$ Informática. RITI Journal, 4(8), 7-13.

RStudio Team (2015). RStudio: Integrated Development for R. RStudio, Inc., Boston, MA. Recuperado de http://www.rstudio.com/

Ruiz, M. A., Pardo, A., \& San Martín, R. (2010) Modelos de Ecuaciones Estructurales. Secciones Monográficas, Papeles del Psicólogo, 31(1), 34-45.

Shook, C., Ketchen, D., Hult, T., \& Kacmar, M. (2004). An Assessment of the Use of Structural Equation Modeling in Strategic Management, Strategic Management Journal, 25(4), 397-404. doi:10.1002/smi.385

Schumacker, R., \& Lomax, R. (2016). A beginner's guide to structural equation modeling. New York: Routledge.

Schmidt, V., Raimundi, M., \& Molina, M. (2015). Satisfacción vital en dominios específicos: adaptación de una escala para su evaluación, Liberabit, 21(2), 299-312.

The WHOQOL Group (1995). The World Health Organization Quality of life assessment (WHOQOL): Position paper from the world health organization. Social Science and Medicine, 41, 143 - 149. doi:10.1016/0277-9536(95)00112-K

Tello, J (2018). Calidad de vida y bienestar psicológico en estudiantes de Psicología de una Universidad Nacional de Lima Metropolitana. (Tesis para optar el título de Licenciado en Psicología), Universidad Nacional Federico Villarreal, Lima, Perú.

Tueros, Y. (2018). Satisfacción familiar y calidad devida en adolescentes de una institución educativa de San Juan de Miraflores. (Tesis para optar el título de Licenciada en Psicología), Universidad Autónoma del Perú, Lima, Perú.

Urzúa, A., \& Caqueo-Urízar, A. (212). Calidad de Vida: Una revisión teórica del concepto. Terapia Psicológica, 3(1), 61-71 doi:10.4067/S0718-48082012000100006

Veenhoven, R. (2000). The four qualities of life. Journal of Happiness Studies, 1(1), 1-39. doi:10.1023/A:1010072010360

Verdam, M. G., Oort, F. J., \& Sprangers, M. A. (2016). Using structural equation modeling to detect response shifts and true change in discrete variables: An application to the items of the SF-36. Quality of Life Research, 25(6), 1361-1383. doi:10.1007/s11136015-1195-0

Williams, B., Onsman, A., \& Brown, T. (2010). Exploratory factor analysis: A five-step guide for novices. Australasian Journal o Paramedicine, 8(3), 1-13. doi:10.33151/ajp.8.3.93

Zapata, G., \& Canet, M.T. (2008). Propuesta metodológica para la construcción de escalas de medición a partir de una aplicación empírica. Revista Electrónica Actualidades Investigativas er Educación, 8(2), 1-26. 